\title{
P2P INFRASTRUCTURE FOR TOURISM ELECTRONIC MARKETPLACE
}

\author{
Luís C. S. Barradas ${ }^{\dagger}$, J. J. Pinto-Ferreira \\ claudio@est.ipcb.pt,jipf@fe.up.pt \\ ${ }^{\dagger}$ E.S.T.-I.P.C.B., Avenida do Empresário, 6000-767, Castelo Branco, PORTUGAL \\ ${ }^{\ddagger}$ FEUP-DEEC / INESC Porto-UESP, Rua Dr. Roberto Frias s/n, 4200-465 Porto, \\ PORTUGAL
}

\begin{abstract}
Internet and information technologies have changed the way people interact and make business all over the world. Unfortunately, this is not completely true in the sense that the fast progress fostered by more dynamic economies has in fact been contributing to widening the so-called digital divide between rich and poor. This is a particularly true for small and medium enterprises in remote communities that face a number of obstacles and difficulties due to their family business structure, limited access to information and communication infrastructure. This paper presents a P2P infrastructure for a distributed $e$ Marketplace that aims at bringing together tourism operators, players of crafts and agro-eco-cultural tourism sectors living in remote communities, in order to provide them the opportunity tojoin, at a reduced cost, to the information society and reach new markets.
\end{abstract}

\section{INTRODUCTION}

Tourism is classically regarded as travelling for recreation, although this definition has been expanded in recent years to include any travel outside of one's normal working or living area. The tourism sector has become a multi-billion international industry, and one that is growing in developed countries at a rate considerably faster than annual growth levels (Wickipedia, 2004). It involves a wide range of industries, like travel agencies industry, transporting industry, hotel trade, etc.

All over the world, touristic offers are organized by countries. These offers are organized by touristic regions that aggregate a wide and assorted range of players of tourism sub-sectors, like eco-tourism, heritage and cultural tourism, crafts, etc. These players are usually small and medium enterprises (SME's) or individuals. However, business opportunities are affected by factors such as country economy, geographic location, enterprise dimension and technological capacity. A craftsman located at the Peru Andes does not have the same opportunities to sell their products as one located at North America or Europe.

This project addresses three tourism sub-sectors, namely crafts, eco- agrotourism and cultural heritage, which are interdependent and complementary for a 
number of activities and practices and strongly linked to declining rural areas. The main goal of this project is therefore to eliminate the digital divide barriers and create equal opportunities and access to the global network where each player of target sector can emerge globally. The business network to be presented in the next paragraphs will enable local communities the access to business collaboration services and give their business global visibility.

\section{ELECTRONIC MARKETPLACES}

The traditional electronic marketplace (e-Marketplace) is a Web portal where buyers and suppliers come together to explore new business opportunities (Ferreira, 2002). Basically, an e-Marketplace brings buyers and sellers together, encouraging them to undertake new trading exchanges, providing a wide range of services to facilitate transactions. At the end, the e-Marketplace entity usually charges a service fee taking the form of a percentage of the transaction value.

E-Marketplaces are an optimal solution, as a start point to buyers and sellers of target sectors to meet each other. However, they need revenue sources to survive, and we need free services and free accesses to guarantee democracy. The solution consists in providing free services (offer publishing, offer look-up, communication between partners, etc) and payable services (e-Banking, business transactions, etc). The critical mass is a key feature of a successful e-Marketplace (Chow, 2000). The speed of growth and the number of users publishing offers and trading should be one of the most important success metrics. However, although traditional eMarketplaces have many advantages, they have also some disadvantages and limitations: they are centralized information repositories; are loosely connected, its members cannot group together or build virtual communities; have price-based decisions and do not allow iterated agreements (Ferreira, 2002). These characteristics point to a number of drawbacks that can ultimately explain why this business model has proved to be ineffective (Ferreira, 2002). On the other hand, Peer-to-Peer (P2P) computing model has allowed the creation of new business models and services that can bring new added value when used in the e-Marketplace construction.

\subsection{Peer-To-Peer Based E-Marketplaces}

The client/server architecture for computing systems was first proposed as an alternative to the conventional mainframe systems approach for large enterprises (Loo, 2003). In a P2P system, computers can act as both clients and servers. Their roles in any task will be determined according to what is most appropriate for the system at the time, which minimizes the workload on servers and maximizes network performance (Barkay, 2002). Many applications were built around this model. Resources shared could be anything: CPU cycles (SETI@home, Folding@home ${ }^{\mathrm{ii}}$ ) or files (Napster ${ }^{\mathrm{iii}}$, Gnutella ${ }^{\mathrm{iv}}$, etc). Peer interactions involve resource advertising, search and subsequent discovery of resources, requests for access to these resources, responses to these requests and exchange of messages between peers (Fox, 2001). This computing model has both technical and social components (Lethin, 2003). It promises freedom from boundaries, censorship, and 
centralized control. Each participant computer or node is called "peer", meaning participants interact as equals (Kubiatowicz, 2003).

Services provided by a $\mathrm{P} 2 \mathrm{P}$ based e-Marketplace can bring numerous advantages. In a P2P network, existing services enable registered suppliers to publish (advertise) information about their offers. Buyers will then use P2P search capabilities to locate and retrieve offers, followed by interaction with selected suppliers over the P2P network infrastructure itself. This new e-Marketplace model overcomes all of the previous disadvantages (Ferreira, 2002). It is more flexible and reinforces trust between business partners.

\section{P2P INFRASTRUCTURE FOR TOURISM E-MARKETPLACE}

Aiming at a fast number of user growth and low cost, it was found adequate that players of the target sectors should have access to a $\mathrm{P} 2 \mathrm{P}$ trading infrastructure providing free basic services such as: offer publishing; business partner look-up; and trading support, by enabling communication between partners. Figure 1 illustrates the proposed scenario, combining the "geo-view" with the corresponding networking infrastructure, a distributed P2P e-Marketplace comprising all the players from various tourism regions.

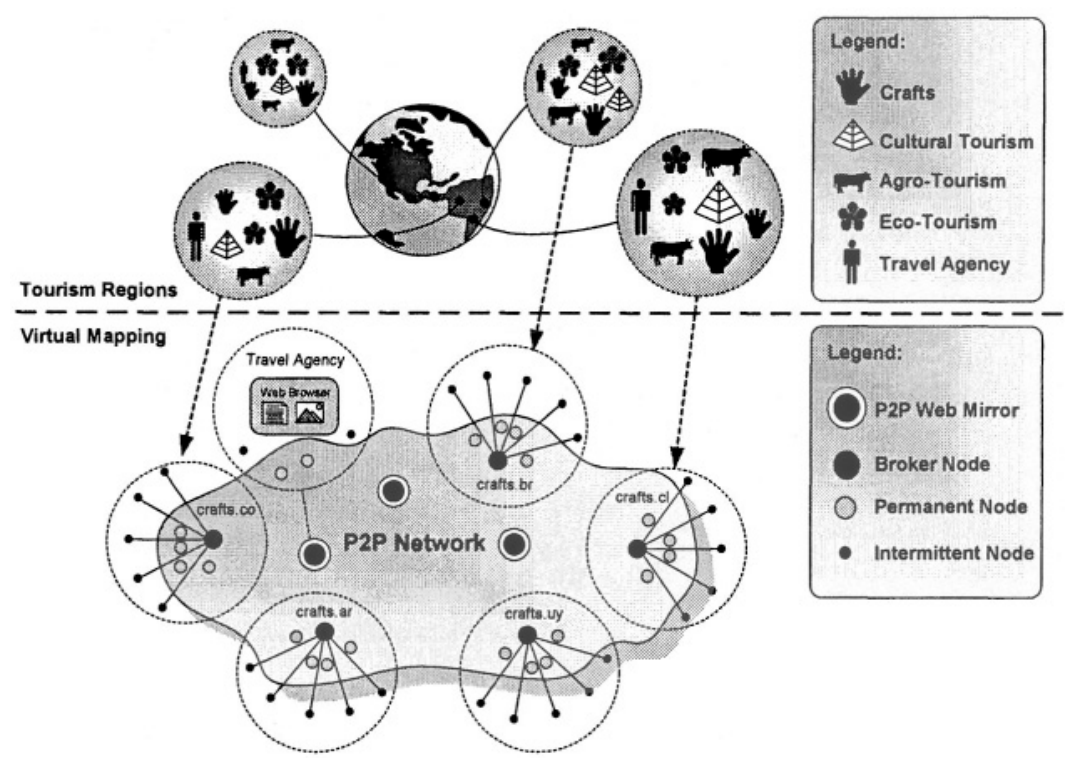

Figure 1 - The P2P Network Infrastructure

The proposed e-Marketplace builds on a P2P network comprising four different node types. The nodes classification is based on the connection type to Internet, function type and the owner's type. The nodes outside the network boundaries don't have a permanent connection to Internet. 


\subsection{P2P Network Architecture}

The e-Marketplace conceptual architecture is illustrated in Figure 1 and comprises the following network nodes: P2P Web Mirror, Broker Node, Permanent Node and Intermittent Node. Each node type is a peer in the network and assumes different roles on the architecture.

P2P network flexibility builds on distribution and look-up flexibility. However, pure P2P look-up services can introduce a huge overhead in network traffic and increase searches latency (Lui, 2002). To tackle this issue, the Web Mirror nodes were conceived in order to aggregate the whole network offer. To this end, a reference index to all offers is kept in these nodes, with offer summary information, each including the address of the peer that provides that offer. As a result of this web mirror service, offer lookup can be made using either pure P2P search mechanisms (slower) or search on the mirror offer database (faster). The node that hosts the P2P Web Mirror also hosts other services, namely the ones that allow for offer management such as combining several touristic offers into one single touristic package, or other services such as electronic payment support. Figure 2a presents the P2P Web mirror architecture. It is a 3-tier based architecture: Interface, Business Logic and Data. Only the architecture's shadowed parts are inherent to this project (the free part). At last, network peers also find each other after logging into the P2P Web mirrors. This means that P2P Web Mirror is also the P2P "rendezvous node".

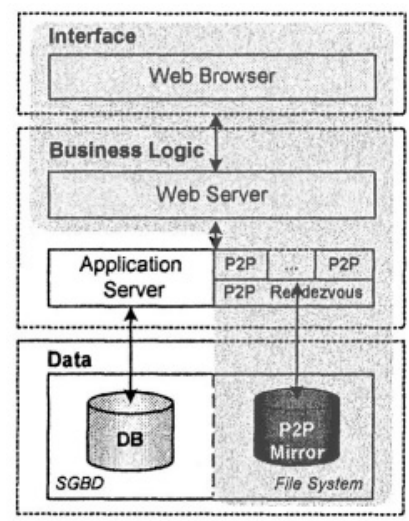

a) P2P Web Mirror

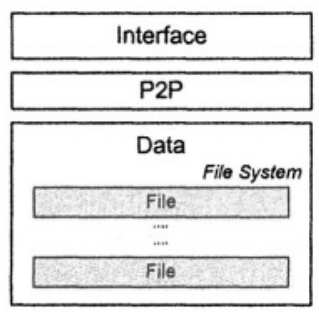

b) Intermittent and Permanent Nodes

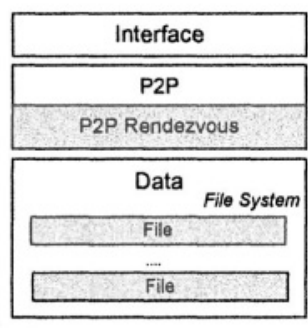

c) Broker Nodes

Figure 2-P2P Network Nodes Architecture

The Web Mirror interface layer is based on a web browser. Through the web browser, the Web Mirrors in the network are the entry point for the users to perform touristic offers' search. The underlying Business Logic layer concerns both the business logic inherent to the e-Marketplace (which is not the emphasis of this work), and the one associated to the P2P mechanisms (e.g.: services providing the means to perform offer lookups in the P2P network). The Data layer comprises the databases related to both e-Marketplace and P2P Network (where offers header information - summary - are kept).

Intermittent nodes are tipically owned by players with economical, technological or geografical limitations. Network accesses are sporadic and supported by links 
with limited bandwidth (e.g.: satellite). Due to these limitations, users must use a broker node to publish their offers. The 3-tier architecture of this node is shown in Figure 2b: Interface, $\mathrm{P} 2 \mathrm{P}$ and data. The interface layer is responsible by an user friendly interface. The P2P layer provides all inherent services to the P2P model. Finally the Data layer, is the local offers repository, whose information is to be uploaded to the broker node whenever a change is made and a connection is available. The local file system is the support base for this layer.

Permanent nodes are owned by players with the capability to maintain a permanent connection to the Internet. Unlike the previous case, these nodes keep their own offers in their file system (Figure $2 b$ ).

Broker nodes keep offers published by intermittent nodes. Its main goal is to guarantee the availability of the intermittent nodes' offers to the peer network. The architecture of the Broker node is similar to the Permanent and Intermitent Node architecture, further including the rendezvous service (Figure 2c).

\subsection{Tourism Offers}

A touristic offer contains the information of about tourism products/services. As an example, a crafts enterprise offer may include information such as artifact name, picture, video, sound, cultural heritage, price and availability. The information contents of each offer is not fixed, but rather adapted to each situation. To ensure this flexibility, offer documents are normalized and structured using XML (eXtended Markup Language). Each offer type will have its own structure. Despite this flexibility, basic summary (header) information will always be the same, thus facilitating its publishing at the Web Mirrors for faster search.

An offer is composed by three documents: a XML Schema that defines the offer structure and its data types; a XML document that contains the offer contents; a XSL (eXtended Stylesheet Language) document that defines the offer presentation. The great advantage of this architecture, is that the offer contents presentation is made using XSLT (XSL Transformations) technology, that allows the direct offer content presentention in a web browser that suports XML. Besides this, it is very simple to create new offer types of other activity sectors. All we have to do, is to define their structure and presentation.

\subsection{Services and Facilities}

\subsubsection{Web Searches Through P2P}

Search is one of the most relevant services in the P2P network. Whenever a tourism player such as a travel agency wants to search touristic offers from a particular country or region, this operation can be made through the web browser by accessing region Web Mirror. The user then has two options: (1) he (she) either searches the local Web Mirror database, updated by all peers whenever they have a new offer or offer update; or (2) using the web interface, or a simple peer-node, the user is granted access to a pure search in the P2P network.

\subsubsection{Establishment of Groups of Nodes}

The P2P model allows the creation of peer groups. A peer group can be seen as set of peers under the same domain (Traversat, 2003). Looking at the target business 
sectors, a group can be seen like an association of target sector player's nodes with common interests (e.g.: crafts association). This concept allows an easy integration and sharing of information inside a particular group or sector ensuring that this information is, for example, invisible to the world outside that group. Figure 3 shows an example of a group of nodes, with a national covering. The member nodes of the group are bounded by the irregular shape.

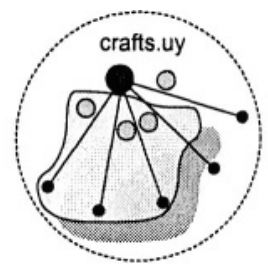

Figure 3 - A Group of Nodes

The groups play an important role in way to improve security, which is an essential issue in the e-business world. Creating secure peer groups increase the trust level between business partners. To join to a secure peer group, a peer needs to present its membership credentials (login and password), which implies that the peer must be a member of that group (Gradecky, 2002). Using asymmetric (e.g.: RSA) or symmetric (e.g.: RC4, DES) encryption algorithms together with hash algorithms (e.g.: MD5), the communications confidentiality and integrity inside the group, are guaranteed.

\subsubsection{Real Time Interactions}

P2P-based e-Marketplace(s) build on services such as web searches through the P2P network, and the ability to obtain extra information about the suppliers. This information can be something like the IP address of the computer that hosts the peer, as well its actual status (on-line/ off-line). Having this information, buyers and suppliers can interact in a simple way. We foresee two types of interactions:

- Synchronous interactions - This interaction type is used when both buyer and supplier are on-line. They can send instant messages to each other, to obtain more information about products, to negotiate and to formalize a deal (e.g.: room reservation at an Agro-tourism farm).

- Asynchronous interactions - This interaction type is used when only one of the parties is on-line. If a buyer wants to communicate to the supplier, he can leave him a message in a virtual mailbox (service similar to e-mail). The message will be delivered to the supplier as soon as he is back on-line.

As needed, messaging can be done by exchanging forms, allowing formal interaction and searching as well as automatic replies by peers. These replies may invoke simple hotel room availability and booking.

Synchronous interactions in particular, are a valuable service for e-Marketplaces, as they can break temporal restrictions for communication, imposed by traditional messaging services as the e-mail, and imposed by the different globe time zones. This can increase speed of transactions between buyers and suppliers. 


\subsection{Illustrating the P2P e-Marketplace}

This sub-section presents an example of two basic services, presenting only two of many possible interactions and configurations that may occur on the network:

- Offer Publishing

- and Searching.

In the example, pictured in Figure 4, the personal computer corresponds to a permanent node in the $\mathrm{P} 2 \mathrm{P}$ network whereas the servers correspond to the $\mathrm{P} 2 \mathrm{P}$ Web Mirror.

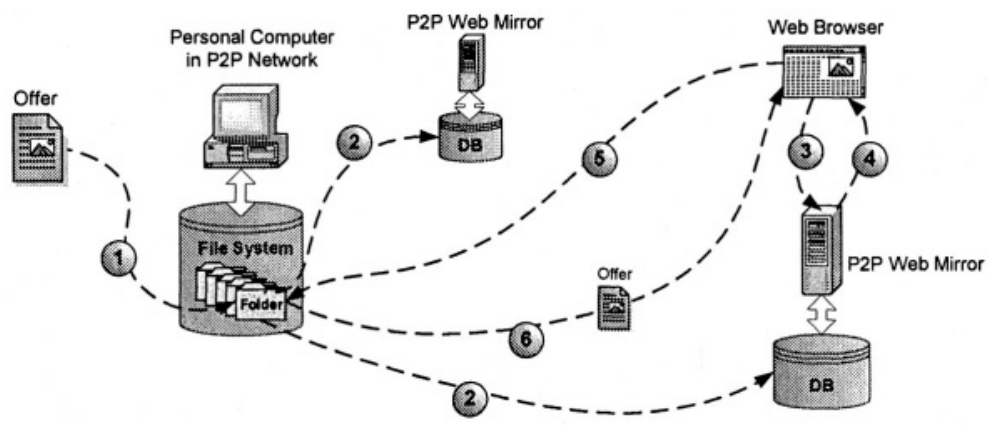

Figure 4 - Offer Publishing and Searching

The offer publishing is a very simple operation. First, a target sector actor publishes his offer, by saving it on his personal computer file system (1). Once published, a broadcast message containing the offer summary (e.g.: Peru Andes Handicraft, Pan flute, etc.) is sent to the P2P web mirrors (2). Mirrors local databases are updated. If a given offer is removed, the event is also broadcasted to $\mathrm{P} 2 \mathrm{P}$ web mirrors, in order to update their databases.

As stated before, the P2P web mirror is the search service entry point on the web. Through a web browser, users can make offer searches (3). The retrieved results of mirror databases are presented in the web browser (4). As soon as the user has it, he can then select it to get full offer details. Getting the full offer detail implies a detailed offer request to the node that owns it (5). This means that user can download the selected offer (6), from its source making a P2P connection to the offer owner node. Once download is completed, user can see the whole offer content on his web browser.

\subsection{Current Status and Future Directions}

This project is yet in an earlier stage of development. The next step will be integration of the P2P infrastructure with an Agent Based Workflow Management System, in way to provide the business processes automation of the target sectors player's organizations, and make them able to support B2B interactions. However, this feature will only makes sense, if applied by tourism enterprises, whose dimension and organizational structure demand for the use of workflow. 


\section{CONCLUSION}

E-Marketplaces are most useful platforms. They lead buyers and sellers to meet each other for trading. In this context, as presented before, traditional e-Marketplace limitations can be overcome by using P2P technology to achieve a solution that is more flexible and reinforces trust among partners. On other hand, P2P computing model promises freedom and Internet equality, where participants interact as equals. Promoting the usage of a P2P infrastructure to support the proposed e-Marketplace seems to be the best strategy to achieve the low cost and the network effect that will ensure the success in reducing the "digital divide", the presented concept:

- Will enable the set up of an e-Marketplace without service charges, where everyone benefits from being able to connect to everyone else;

- Broker nodes guarantee that everyone has a permanent presence on the network, at no added costs;

- Peers can attach to each other and develop virtual communities (groups), creating thus a stronger position in the network and in the value chain;

- The exchange rules can be set by each pair of peers performing an exchange, not by a third party.

Crafts and tourism SME's and related cultural actors have always played an important economic and social role, E-inclusion and the introduction of advanced I\&CT's in these sectors and areas can play a key role in terms of supporting and facilitating local economic development and sustainability.

\footnotetext{
${ }^{\mathrm{i}}$ Seti@home - The Searching of Extraterrestrial Intelligence, http://setiathome.ssl.berkeley.edu

${ }^{\text {ii }}$ Folding@Home Distributed Computing, http://www.stanford.edu/group/pandegroup/folding

iii Napster Inc., http://www.napster.com

${ }^{\text {iv }}$ The Gnutella Protocol, http://www.gnutella.com
}

\section{REFERENCES}

1.Barkai, D. "P2P Computing”, Intel Press, Santa Clara, CA 2002.

2.Chow D, Ghani A, Takeda G, Ziffra S. "Let's forget Vertical! Beyond the B2B Market Hubs - A Study on the Current and Future Trends of B2B Marketplaces", Kellogg TechVenture 2000 Anthology, 2000.

3.Ferreira D, Ferreira JJP. "Building an E-Marketplace on a P2P Infrastructure", in proceedings of 18th International Conference on CAD/CAM, Robotics and Factories of the Future, Porto, 2002.

4.Fox G, Pallickara S. "Peer-to-Peer Interactions in Web Brokering Systems", Ubiquity - ACM IT Magazine and Forum, 2002.

5.Gradecky, Joseph D. "Mastering JXTA - Building Java ${ }^{\mathrm{TM}}$ Peer-to-Peer Applications", Java ${ }^{\mathrm{TM}}$ Open Source Library, Wiley Publishing, 2002, ISBN: 0-471-25084-8.

6. Kubiatowicz, J. "Extracting Guarantees from Chaos", Communications of ACM, Vol. 46, No. 2, pp. 33-38, February 2003.

7.Lethin, R. "Technical and Social Components of Peer-to-Peer Computing", Communications of ACM, Vol. 46, No. 2, pp. 30-32, February 2003.

8. Loo, AW. "The Future of Peer-To-Peer Computing - An economical method for pumping up computing power by tapping into P2P systems using Web server technologies", Communications of ACM, Vol. 46, N.9, September 2003.

9.Lui SM, Kwok SH. "Interoperability of Peer-to-Peer File Sharing Protocols", ACM SIGecom Exchanges, Vol. 3, N.3, August 2002.

10.Traversat, Bernard et all. "Project JXTA 2.0 Super-Peer Virtual Network", May 25, 2003.

11.Wickipedia. "Wikipedia, the free encyclopedia", http://en2.wikipedia.org/wiki/Main_Page. 